\title{
Analyse de l'influence de l'oscillation Arctique sur la variabilité interannuelle des précipitations dans le bassin versant de la rivière Saint-François (Québec, Canada) au moyen de la méthode des corrélations canoniques Influence of the Arctic oscillation on the internannual variability of precipitation in the Saint-François river watershed (Québec, Canada) as determined by canonical correlation analysis
}

\author{
Ali A. Assani, Francis Lajoie, Marie-Ève Vadnais et Guillaume Beauchamp
}

Volume 21, numéro 1, 2008

URI : https://id.erudit.org/iderudit/017928ar

DOI : https://doi.org/10.7202/017928ar

Aller au sommaire du numéro

Éditeur(s)

Université du Québec - INRS-Eau, Terre et Environnement (INRS-ETE)

ISSN

1718-8598 (numérique)

Découvrir la revue

Citer cet article

Assani, A. A., Lajoie, F., Vadnais, M.-È. \& Beauchamp, G. (2008). Analyse de l'influence de l'oscillation Arctique sur la variabilité interannuelle des précipitations dans le bassin versant de la rivière Saint-François (Québec, Canada) au moyen de la méthode des corrélations canoniques. Revue des sciences de l'eau / Journal of Water Science, 21(1), 19-33.

https://doi.org/10.7202/017928ar
Résumé de l'article

Le bassin versant de la rivière Saint-François, situé sur la rive sud (rive droite) du fleuve Saint-Laurent (Québec, Canada), couvre une superficie d'environ $10000 \mathrm{~km}^{2}$. Dans le but de déceler les facteurs climatiques qui influencent les précipitations dans ce bassin versant, nous avons analysé la succession des périodes pluviométriques sèches et humides par la technique des moyennes glissantes sur cinq ans, d'une part, et la relation entre quatre indices climatiques (oscillation arctique, oscillation australe, oscillation nord-atlantique et la température des eaux océaniques de surface) et ces périodes pluviométriques au moyen de l'analyse des corrélations canoniques, d'autre part. Nous avons analysé les données pluviométriques mesurées à trois stations représentatives des régimes pluviométriques du bassin versant : Sherbrooke, Disraeli et Drummondville. Ces données couvrent une période de 76 ans (1914-1990).

En ce qui concerne la variabilité interannuelle des précipitations, nous avons détecté deux types de changement. Le premier type de changement, survenu autour de 1950, concerne la répartition des précipitations à l'échelle du bassin versant (changement spatial). Avant 1950, la succession des périodes sèches et humides des précipitations n'était pas synchrone (opposition des périodes) mais elle l'est devenue après 1950. Le second type de changement a été observé autour des années 1935 et 1970. Il correspond à un changement des totaux pluviométriques au niveau des stations (changement quantitatif). Avant et après 1935 et 1970, on passe ainsi des périodes sèches aux périodes humides ou vice versa selon les stations. En tenant compte de ces trois dates, nous avons observé la succession des périodes sèches et humides suivantes : 1) Avant 1950, entre 1914 et 1935, nous avons observé une période sèche aux stations de Disraeli et de Sherbrooke mais une période humide à la station de Drummondville. Entre 1936-1950, ces périodes se sont inversées : humide à Disraeli et Sherbrooke mais sèche à Drummondville; 2) Après 1950, entre 1951 et 1970, les précipitations étaient déficitaires aux trois stations. En revanche, elles sont devenues excédentaires après 1970.

L'analyse des corrélations canoniques entre les précipitations et les indices climatiques a révélé les faits significatifs suivants : 1) Avant et après 1950, les précipitations sont positivement corrélées à l'oscillation arctique (OA), mais cette corrélation est plus faible après qu'avant 1950. Ainsi, l'augmentation des valeurs de OA entraînerait une hausse de fréquence des masses d'air en provenant du sud dans le bassin versant; 2) Lorsqu'on considère les périodes sèches et humides, $\mathrm{OA}$ est toujours positivement corrélée aux périodes sèches à la station de Sherbrooke. 


\title{
ANALYSE DE L'INFLUENCE DE L'OSCILLATION ARCTIQUE SUR LA VARIABILITÉ INTERANNUELLE DES PRÉCIPITATIONS DANS LE BASSIN VERSANT DE LA RIVIÈRE SAINT-FRANÇOIS (QUÉBEC, CANADA) AU MOYEN DE LA MÉTHODE DES CORRÉLATIONS CANONIQUES
}

\author{
Influence of the Arctic oscillation on the internannual variability of precipitation \\ in the Saint-Francois river watershed (Québec, Canada) as determined by canonical correlation analysis
}

Ali A. ASSANI*, Francis LAJOIE, Marie-Ève VADNAIS, Guillaume BEAUCHAMP

Laboratoire d'hydro-climatologie et de géomorphologie fluviale, section de géographie, Pavillon Léon-Provencher, Université du Québec à Trois-Rivières, 3351, boulevard des Forges, Québec, G9A 5H7, Canada

Reçu le 22 mars 2006, accepté le 4 mai 2007

\section{RÉSUMÉ}

Le bassin versant de la rivière Saint-François, situé sur la rive sud (rive droite) du fleuve Saint-Laurent (Québec, Canada), couvre une superficie d'environ $10000 \mathrm{~km}^{2}$. Dans le but de déceler les facteurs climatiques qui influencent les précipitations dans ce bassin versant, nous avons analysé la succession des périodes pluviométriques sèches et humides par la technique des moyennes glissantes sur cinq ans, d'une part, et la relation entre quatre indices climatiques (oscillation arctique, oscillation australe, oscillation nord-atlantique et la température des eaux océaniques de surface) et ces périodes pluviométriques au moyen de l'analyse des corrélations canoniques, d'autre part. Nous avons analysé les données pluviométriques mesurées à trois stations représentatives des régimes pluviométriques du bassin versant: Sherbrooke, Disraeli et Drummondville. Ces données couvrent une période de 76 ans (1914-1990).
En ce qui concerne la variabilité interannuelle des précipitations, nous avons détecté deux types de changement. Le premier type de changement, survenu autour de 1950, concerne la répartition des précipitations à l'échelle du bassin versant (changement spatial). Avant 1950, la succession des périodes sèches et humides des précipitations n'était pas synchrone (opposition des périodes) mais elle l'est devenue après 1950. Le second type de changement a été observé autour des années 1935 et 1970 . Il correspond à un changement des totaux pluviométriques au niveau des stations (changement quantitatif). Avant et après 1935 et 1970, on passe ainsi des périodes sèches aux périodes humides ou vice versa selon les stations. En tenant compte de ces trois dates, nous avons observéla succession des périodes sèches et humides suivantes : 1) Avant 1950, entre 1914 et 1935, nous avons observé une période sèche aux stations de Disraeli et de Sherbrooke mais une période humide à la station de Drummondville. Entre 1936-1950, ces périodes se sont inversées : humide à Disraeli et Sherbrooke mais sèche à Drummondville; 2) Après 1950,

*Auteur pour correspondance

Téléphone: 819-376-5011

Télécopie: 819-376-5179

Courriel : ali.assani@uqtr.ca 
entre 1951 et 1970, les précipitations étaient déficitaires aux trois stations. En revanche, elles sont devenues excédentaires après 1970.

L'analyse des corrélations canoniques entre les précipitations et les indices climatiques a révélé les faits significatifs suivants : 1) Avant et après 1950, les précipitations sont positivement corrélées à l'oscillation arctique (OA), mais cette corrélation est plus faible après qu'avant 1950. Ainsi, l'augmentation des valeurs de OA entraînerait une hausse de fréquence des masses d'air en provenant du sud dans le bassin versant; 2) Lorsqu'on considère les périodes sèches et humides, $\mathrm{OA}$ est toujours positivement corrélée aux périodes sèches à la station de Sherbrooke.

\section{Mots clés : Précipitations totales annuelles, oscillation arc- tique, moyennes mobiles simples, analyse des corrélations canoniques, Saint-François, Québec.}

\section{SUMMARY}

The Saint-François River watershed, located on the south shore of the St. Lawrence River (Québec, Canada), covers an area of about $10,000 \mathrm{~km}^{2}$. To detect the climatic factors that influence precipitation in this watershed, we analyzed the succession of dry and wet pluviometric periods by a method of simple moving averages computed over five years. In addition, the relationship between four climatic indices (Arctic Oscillation, Southern Oscillation, North Atlantic Oscillation and Sea Surface Temperature) and these pluviometric periods was analyzed by means of canonical correlation analysis. We analyzed the pluviometric data measured over a 76-year period (1914-1990) at three stations representative of the watershed's pluviometric regimes: Sherbrooke, Disraeli and Drummondville.

Two types of change in the inter-annual variability of precipitation were detected. The first type of change, occuring circa 1950, concernend the distribution of precipitation throughout the watershed, i.e. spatial change. Before 1950, the succession ofdry and wet precipitation periods was asynchronous (opposition of periods), but became synchronous after 1950 . The second type of change, corresponding to a change in the pluviometric totals at the stations, i.e. quantitative change, was observed circa 1935 and 1970. There was, therefore, a shift from dry to wet periods or vice versa, prior to and following 1935 and 1970, depending on the station. By accounting for these three dates, we observed the succession of the following dry periods and wet periods. First, before 1950 and between 1914 and 1935, we observed a dry period at the Disraeli and
Sherbrooke stations and a wet period at the Drummondville station. Between 1936 and 1950, these periods were reversed: wet periods at Disraeli and Sherbrooke but a dry period at Drummondville. Second, after 1950 and between 1951 and 1970, there was a precipitation deficit at all three stations, which, however, moved into a surplus phase after 1970.

The canonical correlation analysis of precipitation levels and the climate indices revealed the following significant facts: 1) prior to and following 1950, precipitation was positively correlated to the Arctic Oscillation (AO) indices, but this correlation was weaker after 1950 than before; and 2) with respect to the wet and dry periods, the AO index is still positively correlated with the dry periods at the Sherbrooke station.

Key words: annual precipitations, arctic oscillation, moving average, canonical correlation analysis, St-François, Québec.

\section{INTRODUCTION}

Il est de plus en plus admis que l'oscillation arctique, connue aussi sous le nom du mode annulaire de l'hémisphère Nord, est le principal facteur climatique expliquant la variabilité interannuelle du climat en Amérique du nord (AMBAUM et al., 2001; BALDWIN et DUNKERTON, 1999; DESER C., 2000; HIGGINS et al., 2000; RIGOR et al., 2002; THOMPSON et WALLACE, 1998, 2001, WALLACE, 2000; WALLACE et THOMPSON, 2002). Cependant, la plupart des travaux consacrés à la partie Nord-Orientale du continent nord américain (le Nord-Est et l'Est du Canada ainsi que le Nord-Est des États-Unis) se sont surtout intéressés à l'analyse de l'influence de ce facteur sur la variabilité interannuelle des débits. Ainsi, au Nord-Est et à l'Est du Canada, les débits de rivières sont négativement corrélés à l'oscillation arctique (ANCTIL et COULIBALY, 2004; COULIBALY et BURN, 2004, 2005; DÉRY et WOOD, 2004, 2005; DÉRY et al., 2005; KINGSTON et al., 2006) alors que dans la région NordOrientale des États-Unis d'Amérique, les deux variables sont positivement corrélées (KINGSTON et al., 2006). Toutefois, au Québec (au sud du $50^{\circ} \mathrm{N}$ ), la relation entre l'oscillation arctique et les débits est relativement complexe et n'est pas constante dans le temps (ANCTIL et COULIBALY, 2004). En effet, avant 1970, cet indice climatique fut négativement corrélé aux débits de rivières de la rive Nord mais positivement corrélé aux débits de celles de la rive Sud. Mais après cette date, il devient négativement corrélé aux rivières de deux rives. Enfin, aussi bien au Québec que dans le reste du Canada, les valeurs des coefficients de corrélation étaient plus élevées avant 
qu'après 1950 (ANCTIL et COULIBALY, 2004; COULIBALY et BURN, 2004).

En ce qui concerne les éléments climatiques, SHABBAR et BONSAL (2004) ont observé que les hivers caractérisés par des valeurs positives (élevées) d'oscillation arctique sont associés à une fréquence et à une durée nettement plus grandes des périodes de temps froid dans l'Est du Canada. En revanche, à de nombreux endroits des États-Unis, les valeurs positives de cet indice en hiver sont généralement associées à une plus grande fréquence des périodes de temps chaud (HIGGINS et al., 2000). Quant aux précipitations, DÉRY et WOOD (2004) ont observé une corrélation significative négative entre les précipitations et l'oscillation arctique dans le Nord-Est du Canada. En revanche, cette corrélation devient positive dans la région Nord-Est des États-Unis d'Amérique (KINGSTON et al., 2006).

Il ressort de ces études que l'influence de l'oscillation arctique sur les variables hydro-climatiques n'est pas uniforme dans la partie Nord-Est du continent nord américain. Dans le Nord-Est et l'Est du Canada, les phases positives de l'oscillation arctique sont associées à une diminution des précipitations et des débits alors que dans la région du Nord-Est des États-Unis d'Amérique, cette relation s'inverse.

Dans la partie méridionale du Québec (au sud du 50 parallèle Nord), aucune étude n'existe encore sur l'influence de l'oscillation arctique sur la variabilité interannuelle des précipitations. Les études précédentes se sont surtout intéressées à l'analyse de l'influence des autres indices climatiques, tels que l'oscillation australe, l'oscillation nord-atlantique et l'oscillation nord Pacifique (BROWN et GOODISON, 1996; GROISMAN et EASTERLING, 1994; SHABBAR et al., 1997; STONE et al., 2000). Il était donc nécessaire de combler cette lacune dans la mesure où ANCTIL et COULIBALY (2004) ont mis en évidence la complexité de la relation entre l'oscillation arctique et les débits dans cette région du Québec. Par ailleurs, l'existence d'une corrélation significative entre un indice climatique et les débits n'implique pas automatiquement une corrélation significative entre cet indice et les précipitations. En effet, en Nouvelle-Angleterre (États-Unis) par exemple, BRADBURY et al. (2002) ont observé une corrélation significative entre les débits et l'oscillation nord-atlantique alors que celleci n'était pas significativement corrélée aux précipitations. Il est donc important de vérifier si l'oscillation arctique est significativement corrélée aux précipitations au sud du $50^{\circ} \mathrm{N}$ au Québec malgré l'existence d'un lien significatif entre cet indice climatique et les débits. De plus, en cas d'un lien significatif, il est aussi intéressant de déterminer si ce lien demeure constant dans le temps.
Pour vérifier cette hypothèse, nous avons choisi d'analyser les précipitations dans le bassin versant de la rivière SaintFrançois en raison de l'existence des données de mesure de longue durée à plusieurs stations. De plus, ce bassin versant a été particulièrement confronté à des inondations récurrentes durant la décennie 70 (JONES, 2004; SAINTLAURENT et al., 2001). Ces inondations ont été attribuées principalement à la quantité des précipitations. Ainsi, il apparaît important de déterminer si ces inondations pouvaient être reliées à l'oscillation arctique. Dans cette perspective, cette étude poursuit les deux objectifs suivants :

- Analyser la succession des périodes pluviométriques sèches et humides. C'est cette succession qui permet de mieux rendre compte de l'intensité et de la fréquence des inondations dans un bassin versant. De fait, l'objectif est de déterminer si l'occurrence des périodes humides et sèches des précipitations est synchrone dans tout le bassin versant de la rivière Saint-François durant le $\mathrm{XX}^{\mathrm{e}}$ siècle. Il va sans dire que dans cette étude, la question de la stationnarité des séries pluviométriques ne sera pas abordée. Par ailleurs, la connaissance de cette succession des périodes humides et sèches est importante en géomorphologie fluviale car elle permet de suivre les changements qui affectent l'évolution morphologique des chenaux (recoupement des méandres, élargissement et/ou incision des chenaux). Ces changements ne sont pas toujours associés à une hausse ou à une baisse significative (absence de stationnarité) des débits.

- Comparer l'influence de l'oscillation arctique à celle des autres indices climatiques sur cette succession de périodes sèches et humides afin de pouvoir prédire éventuellement les périodes sèches et humides à partir de cette oscillation. Étant donné que le bassin versant est situé dans une région de transition entre l'Est du Canada et le NordEst des États-Unis d'Amérique, il est intéressant de déterminer si la corrélation entre l'oscillation arctique et les précipitations est négative (Est du Canada) ou positive (Nord-Est des États-Unis).

\section{MÉTHODOLOGIE}

\subsection{Choix des stations d'étude et sources de données}

La rivière Saint-François, un des affluents principaux du fleuve Saint-Laurent en rive Sud, couvre une superficie d'environ $10000 \mathrm{~km}^{2}$. L'altitude du bassin versant varie entre 14 et $1067 \mathrm{~m}$., mais plus de $60 \%$ du bassin versant sont situés entre 200 et $500 \mathrm{~m}$ d'altitude. La physiographie du bassin versant de la rivière Saint-François est constituée 


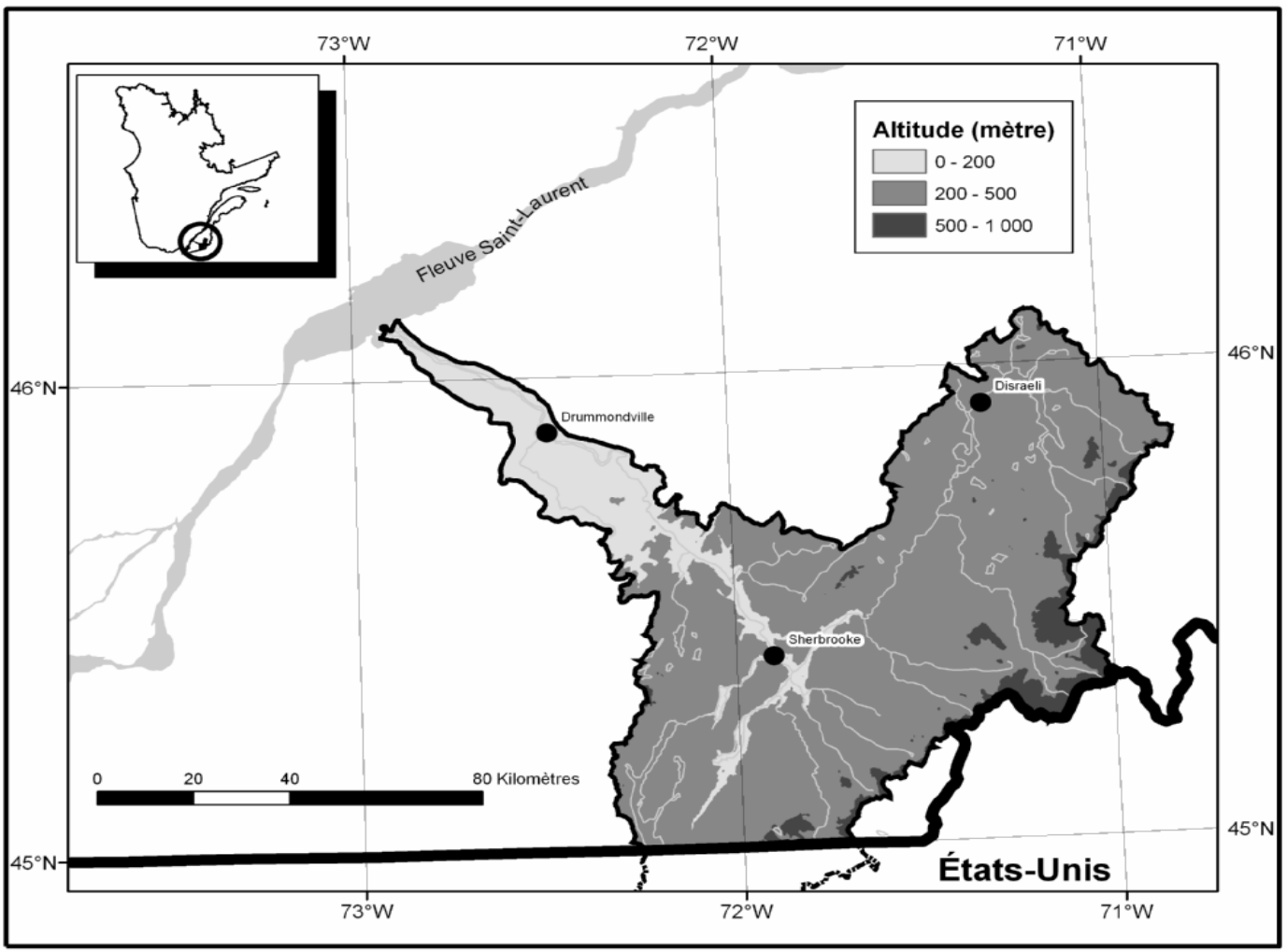

Figure1. Localisation des stations pluviométriques analysées.

Location of pluviometric stations.

de deux unités topographiques distinctes : les Basses-Terres, dans la partie inférieure du bassin versant, et le Haut Plateau Appalachien, au centre et dans la partie supérieure du bassin versant (Figure 1). Ce dernier est caractérisé par un relief de collines et de montagnes occupées surtout par des terres forestières ou des terres en pâturage, alors que la région aval faisant partie des Basses-Terres est caractérisée par une topographie plane ou subhorizontale où dominent surtout les activités agricoles et urbaines. Ces deux unités topographiques correspondent chacune à une région pluviométrique homogène (SIEW-YAN-YU et al., 1998).

Il règne dans le bassin versant un climat tempéré continental atténué par l'influence maritime. Les totaux pluviométriques annuels varient entre 1000 à $1300 \mathrm{~mm}$. Les précipitations totales moyennes les plus élevées sont enregistrées dans le Haut Plateau Appalachien (PROULX et al., 1987). Le bassin versant est balayé par des masses d'air d'origines différentes : les masses d'air du Golfe de Mexique qui proviennent du Sud-Ouest et les masses d'air polaires, du Nord-Ouest et du Nord, ainsi que les masses d'air de l'Océan Atlantique de circulation Ouest et Sud-Ouest (SIEW-YAN-YU et al., 1998). Selon Bousquet (cité par SIEW-YAN-YU et al., 1998), ce sont les masses d'air d'Ouest et du Sud-Ouest qui apportent la plus grande quantité des précipitations en été dans la région. Par contre, en hiver, ce sont des masses d'air du secteur Nord-Est (NNE et ENE) qui sont à l'origine d'une grande partie des précipitations (SIEW-YAN-YU et al., 1998).

Dans chacune de ces deux régions pluviométriques homogènes, nous avons choisi une station dont les mesures des précipitations s'étendaient sur une période d'au moins 50 ans. Ce choix se justifie du fait que nous avons supposé que, dans une région pluviométrique homogène, la variabilité interannuelle des précipitations est aussi homogène. Par conséquent, dans chaque région pluviométrique homogène, l'analyse de la variabilité interannuelle des précipitations à une seule station peut suffire. Ainsi, nous avons retenu les stations suivantes: Sherbrooke (région du Haut Plateau Appalachien) et Drummondville (région des Basses-Terres). Toutefois, le bassin versant est orienté selon deux principaux axes (Figure 1) : Nord-Sud et Est-Ouest. Pour tenir compte de ces deux axes, nous avons ajouté une troisième station (Disraeli), située au nord de la station de Sherbrooke. Ainsi, les trois stations représentent les deux principales extensions du bassin versant. L'axe Est-Ouest est représenté par les stations de Sherbrooke et de Drummondville et celui Nord-Sud, par les stations de Sherbrooke et de Disraeli.

Pour pouvoir comparer l'influence de l'oscillation arctique sur la variabilité interannuelle des précipitations, nous avons analysé les données des précipitations mesurées sur une 
période commune qui s'étend de 1914 à 1990, soit 76 ans. Les mesures des précipitations ont débutéen 1914 à la station de Drummondville et se sont arrêtées en 1990 à la station Disraeli. Quoi qu'il en soit, la période analysée est représentative de la variabilité des précipitations durant le $\mathrm{XX}^{\mathrm{e}}$ siècle dans le bassin versant. Les données des précipitations proviennent des données climatiques d'Environnement Canada (ENVIRONNEMENT CANADA, 2003).

Quant aux indices climatiques, outre l'Oscillation Arctique (OA), nous en avons retenu trois autres : Oscillation Nord-Atlantique (ONA), Oscillation Australe (OAU) et la température des eaux de surface océanique mesurée dans la région Nino3 (NIN3). Ces quatre indices sont abondamment décrits dans la littérature. Leur influence sur la variabilité du climat en Amérique du Nord, en général, et au Québec, en particulier, a été déjà aussi analysée par de nombreux auteurs (ANCTIL et COULIBALY, 2004; BROWN et GOODISON, 1996; CADET et GARNIER, 1988 ; COULIBALY et BURN, 2004, 2005; DÉRY et WOOD, 2004, 2005; ROGERS, 1984; ROPELEWSKI et HALPERT, 1987; SHABBAR et al., 1997a, 1997b; SHABBAR A. et W. SKINNER, 2004; SHERIDAN, 2002, 2003; THOMPSON et WALLACE, 1998; 2001; WANNER, 1999). Nous n'insisterons pas beaucoup sur leur description (Tableau 1). Les données de ces indices ont été tirées du site internet de la NOAA à l'adresse suivante : http://www.cpc.ncep.noaa.gov/ data (consulté en juin 2005). À l'échelle annuelle, nous avons calculé les moyennes annuelles à partir des valeurs mensuelles. Pour chaque année, à l'échelle saisonnière, nous avons calculé deux types de moyennes : les moyennes semestrielles hivernales (d'octobre à mars) et estivales (d'avril à septembre).

\subsection{Analyse statistique de données}

L'analyse statistique de données a été effectuée en deux étapes :

- La première étape a consisté à lisser les séries pluviométriques et les indices climatiques au moyen de la technique des moyennes glissantes calculées sur cinq ans. En ce qui concerne les précipitations, le lissage a été appliqué aux séries standardisées (centrées et réduites) car les séries des indices climatiques le sont déjà. Cette méthode, suggérée entre autres par DÉRY et al. (2005), poursuit trois objectifs : i) mettre en évidence la succession des périodes sèches et humides; ii) maximiser les valeurs des coefficients de corrélation calculées entre les variables en éliminant les fluctuations annuelles des précipitations qui ont tendance à diminuer les valeurs des coefficients de corrélation; et enfin, iii) pouvoir comparer aisément et simultanément l'intensité des périodes sèches et humides de plusieurs stations (ASSANI, 1999). Ces trois objectifs

Tableau 1. Description sommaire des quatre indices climatiques utilisés.

Table 1. Summary of the four climatic oscillations.

\begin{tabular}{lll}
\hline \multicolumn{1}{c}{ Indices } & Localisation du phénomène & \multicolumn{1}{c}{ Mode de calcul des indices } \\
\hline Oscillation Arctique (OA) & $\begin{array}{l}\text { Hémisphère nord extratropicale } \\
\left(20^{\circ}-90^{\circ} \mathrm{N}\right)\end{array}$ & $\begin{array}{l}\text { Valeurs de la première composante } \\
\text { principale du champ de pression en } \\
\text { hiver dans l'hémisphère nord }\end{array}$ \\
Oscillation Nord Atlantique (ONA) & Zone nord-Atlantique extratropicale & $\begin{array}{l}\text { Différence de pressions entre le sud } \\
\text { (Ponta Delgada en Espagne) et le } \\
\text { nord (Reykjavik en Islande) du bassin } \\
\text { Atlantique-Nord }\end{array}$ \\
Oscillation Australe (AUS) & Océan austral tropical & $\begin{array}{l}\text { Différence de pressions entre le } \\
\text { Pacifique austral occidental (Darwin) et } \\
\text { le Pacifique austral central (Tahiti) }\end{array}$ \\
& $\begin{array}{l}\text { Pacifique tropical oriental } \\
\text { (5N-5S, 150W-90W) }\end{array}$ & $\begin{array}{l}\text { Anomalies de la température des eaux } \\
\text { de surface océanique. }\end{array}$ \\
\hline
\end{tabular}


ne peuvent pas être atteints par d'autres méthodes comme, entre autres, l'analyse des ondelettes, qui est de plus en plus utilisée (LABAT, 2005).

- La seconde étape a consisté en l'analyse des corrélations entre les séries lissées standardisées des indices climatiques (groupe des variables indépendantes) et des précipitations mesurées aux trois stations (groupe des variables dépendantes) au moyen de l'analyse des corrélations canoniques. Cette technique présente l'avantage d'analyser simultanément les deux groupes de variables dépendantes et indépendantes. Elle permet de mettre ainsi en évidence les liens entre les variables du même groupe, d'une part, et ceux qui existent entre les variables de deux groupes, d'autre part. De plus, cette méthode permet de maximiser les coefficients de corrélation entre les deux groupes de variables. La méthode de l'analyse canonique des corrélations est exposée dans de nombreux livres de statistiques (AFIFI et CLARK, 1996). Elle est de plus en plus utilisée en climatologie pour analyser la relation entre les variables (température, précipitations, vents, etc.) et les indices climatiques (e.g. BERRI et BERTOSA, 2004; CHEN et CHEN, 2003; DÜNKELOH et JACOBEIT, 2003; HAYLOCK et GOODESS, 2004; JAIN et al., 2005; LOLIS et al., 2004).

\section{RÉSULTATS}

\subsection{Comparaison de la variabilité interannuelle des précipitations annuelles de trois stations}

La variabilité interannuelle des précipitations est présentée sur la figure 2. Après standardisation et lissage de ces données brutes, cette variabilité est caractérisée par une alternance des périodes sèches (valeurs inférieures à la moyenne) et humides (valeurs supérieures à la moyenne) (Figure 3). À la station de Sherbrooke, cette variabilité est caractérisée par l'alternance de deux périodes sèches (1914-1933 et 1953-1970) et de deux périodes humides (1935-1954 et 1970-1985). Quant à la station de Disraeli, elle a connu une très longue période du déficit pluviométrique jusqu'en 1970, à l'exception d'une période relativement humide entre 1946 et 1953. À Drummondville, une longue période de sécheresse survenue entre 1931 et 1970 sépare deux périodes humides (1914-1933 et 1970-1983). Ainsi, les périodes sèches et humides ont tendance à durer plus longtemps à Drummondville qu'aux deux autres stations. Notons aussi que la quantité des précipitations était plus faible durant la seconde que durant la première période humide. Mais elle fut plus faible aussi que celle enregistrée aux deux autres stations durant la décennie 70 .
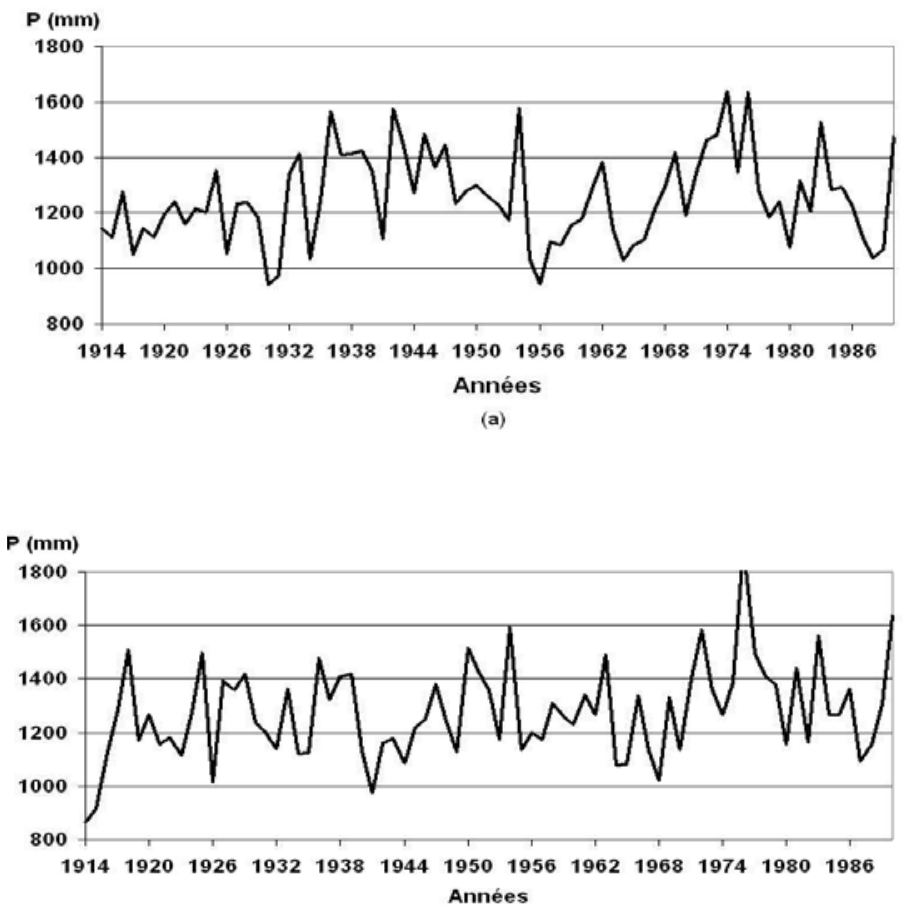

(b)

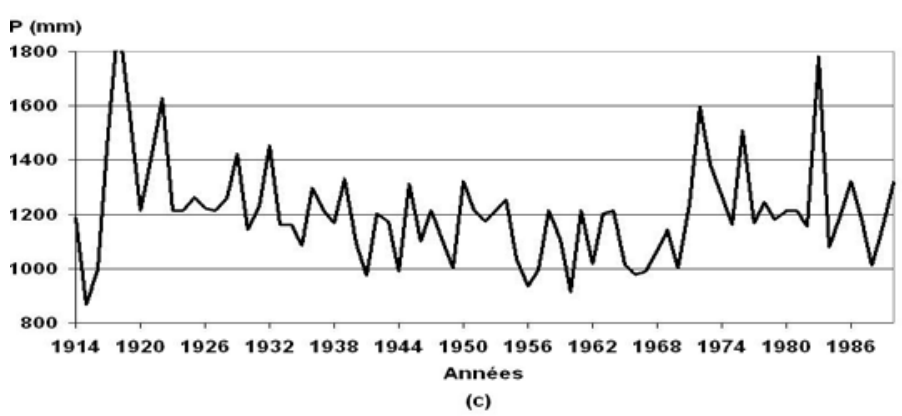

Figure 2. Variabilité interannuelle des précipitations aux stations de Sherbrooke (a), Disraeli (b) et de Drummondville (c) pendant la période 1914-1990.

Inter-annual variability of annual precipitations at the Sherbrooke (a), Disraeli (b) and Drummondville (c) stations (1914-1990).

Si on tient compte de toutes les stations, on peut distinguer deux types de changements :

- Le premier type de changement est survenu autour de 1950. Il affecte la répartition des précipitations dans le bassin versant. En effet, avant 1950, la succession des périodes sèches et humides n'y est pas synchrone. Il existe donc une opposition dans la succession de ces périodes. Cette opposition est surtout évidente entre les stations de Sherbrooke et de Drummondville (Figure 3), c'est-àdire selon l'axe Est-Ouest. La période sèche à Sherbrooke correspond à la période humide à Drummondville et vice versa. Après 1950, la succession de ces périodes sèches et humides devient synchrone aux trois stations. 


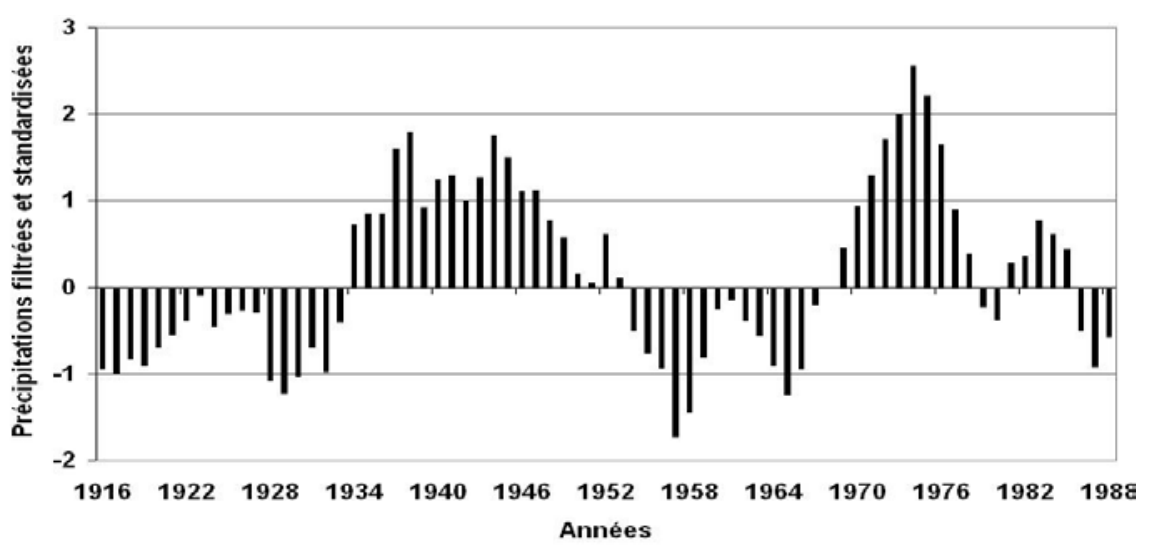

(a)

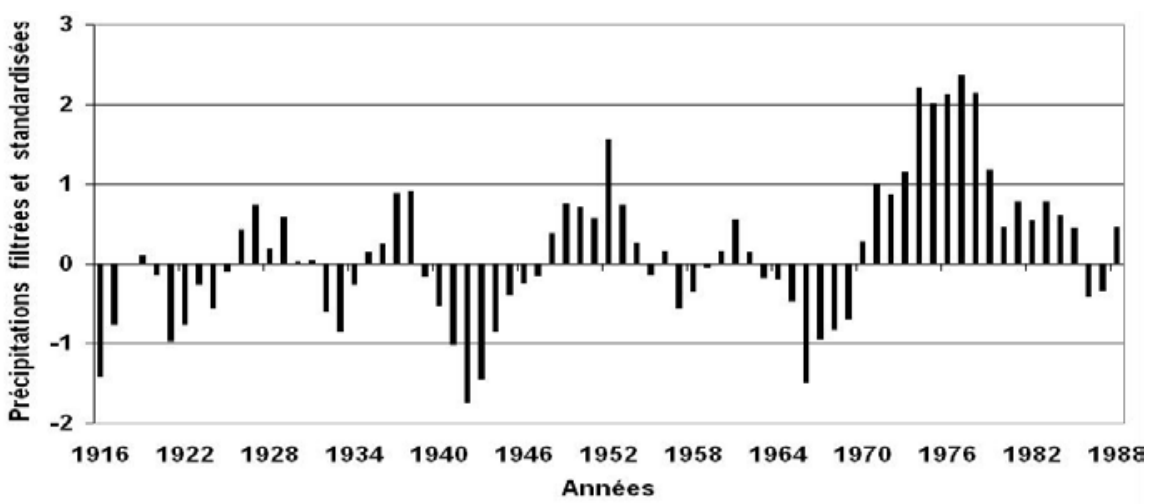

(b)

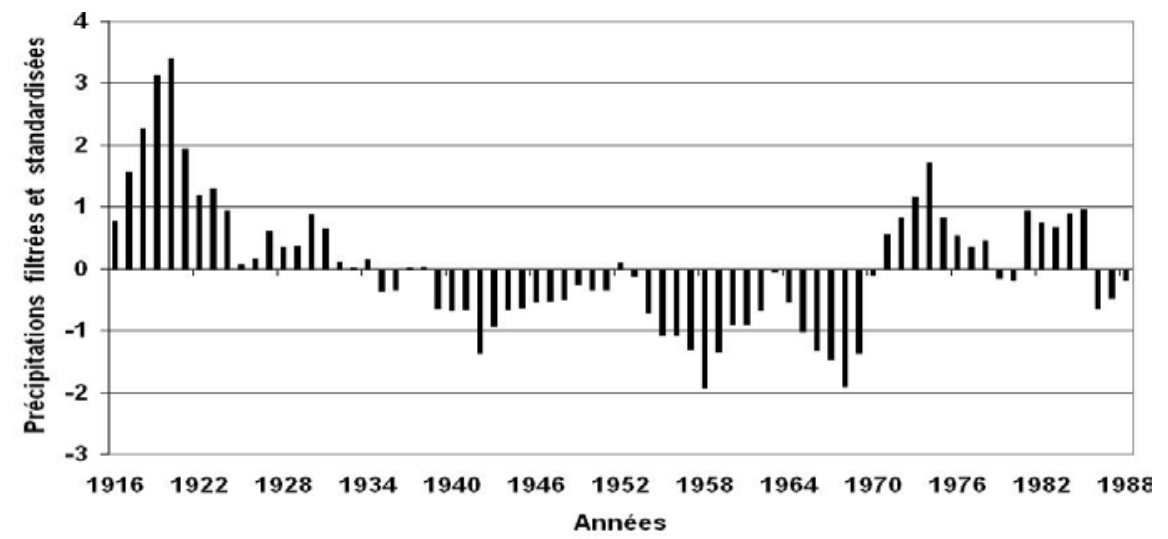

(c)

Figure 3. Succession des périodes sèches et humides aux stations de Sherbrooke (a), de Disraeli (b) et de Drummondville (c) pendant la période 1914-1990. Moyennes mobiles pondérées et standardisées calculées sur cinq ans.

Sequence of dry and wet periods (normalized and five-year running means of the annual precipitation values) at the Sherbrooke (a), Disraeli (b) and Drummondville (c) stations (1914-1990).

- Le second type de changement est survenu autour des années 1935 et 1970 . Il correspond à un changement des totaux pluviométriques dans les trois stations. Dans le cas de la station de Sherbrooke, avant 1935, les précipitations étaient déficitaires alors qu'elles sont devenues excédentaires après cette date. Ce fut l'inverse à la station de Drummondville. Avant 1970, une sécheresse a sévi dans tout le bassin versant suivi d'une période pluvieuse après cette date.

Nous allons maintenant analyser les indices climatiques susceptibles de rendre compte de ces deux types de changements qui affectent la variabilité interannuelle des 
précipitations dans le bassin versant de la rivière SaintFrançois.

\subsection{Relation entre les précipitations annuelles et les indices climatiques pendant la période 1914-1990}

Les valeurs de trois coefficients de corrélation canonique extraites des deux groupes des variables sont toutes significatives (Tableau 2). La valeur du premier coefficient de corrélation canonique, qui mesure le degré de liaison entre les deux groupes de variables, est supérieure à 0,900. Elle exprime ainsi un lien relativement élevé entre les précipitations et les indices climatiques dans le bassin versant de la rivière Saint-François. En ce qui concerne le lien entre les stations et les nouvelles variables canoniques, le tableau 3 révèle que chaque station est corrélée à une nouvelle variable canonique. Ainsi, la station de Sherbrooke est corrélée à la première variable canonique (V1) et les deux autres stations de Disraeli et de Drummondville sont corrélées respectivement aux variables canoniques V3 et V2. Ces coefficients de corrélation sont positifs. Quant aux indices climatiques, la première variable canonique (W1) est fortement corrélée à l'indice semestriel estival de l'oscillation arctique (OA), la seconde nouvelle variable canonique (W2) est significativement corrélée à l'indice semestriel estival de l'oscillation nord-atlantique (ONA). Mais cette corrélation est négative. Quant à la dernière variable canonique (W3), elle est positivement corrélée aux deux oscillations (ONA et $\mathrm{OA}$ ) mais cette corrélation est plus élevée avec ONA. Il ressort de ces résultats que l'oscillation arctique $(\mathrm{OA})$ est significativement corrélée aux stations de Sherbrooke et de Drummondville (axe Est-Ouest) alors que l'oscillation nord-atlantique, considérée comme la composante régionale de $\mathrm{OA}$, l'est aux stations du
Drummondville et de Disraeli. OA est positivement corrélée aux deux stations. En revanche, ONA est négativement corrélée à Disraeli en été mais positivement corrélée à Drummondville en hiver. Son influence dépend des saisons. Enfin, on retiendra que les indices hivernaux de OA et ONA sont corrélés à la troisième nouvelle variable canonique. Par conséquent, c'est durant cette saison que les deux indices deviennent corrélés comme l'avait déjà mentionné WALLACE (2000). La comparaison de la variabilité interannuelle de ces indices révèle que leur variabilité devient quasi synchrone après 1950 (Figure 4).

\subsection{Relation entre les précipitations annuelles et les indices climatiques avant et après 1950}

Pour les deux périodes, les valeurs des premiers coefficients de corrélation canonique sont plus élevées que pour l'ensemble de toute la période (Tableau 4). Le premier coefficient de corrélation canonique de la période avant 1950 est plus élevé que celui de la période après 1950 . En revanche, avant la période 1950, seuls les deux premiers coefficients de corrélation canonique sont significatifs alors qu'après cette date, tous les coefficients de corrélation canonique le sont.

En ce qui concerne la relation entre les nouvelles variables canoniques et les précipitations mesurées aux trois stations, il ressort du tableau 5 qu'avant la période 1950, les stations de Drummondville et de Sherbrooke sont fortement corrélées à la première variable canonique; la première station étant corrélée négativement et la seconde, positivement. En effet, cette opposition confirme celle observée dans la succession des périodes sèches et humides aux deux stations : l'épisode sec à

Tableau 2. Les coefficients de corrélation canonique calculés entre les trois stations (Disraeli, Drummondville et Sherbrooke) et les indices climatiques annuels et semestriels (OA, ONA, AUS et Nino.3) dans le bassin versant de la rivière Saint-François (1914-1990).

Table 2. Canonical correlation coefficients between precipitation and climatic oscillations (annual and alf-annual) in Saint-François River watershed (1914-1990).

\begin{tabular}{lcccc}
\hline & $\mathbf{R}$ & Valeur propre & Valeur de $\mathbf{F}$ & pr $>\mathbf{F}$ \\
\hline$\lambda_{1}$ & 0,9177 & 5,339 & $10,85(36,172)$ & $<0,0001$ \\
$\lambda_{2}$ & 0,7975 & 1,747 & $6,90(22,118)$ & $<0,0001$ \\
$\lambda_{3}$ & 0,6887 & 0,902 & $5,41(10,60)$ & $<0,0001$ \\
\hline
\end{tabular}

$\mathrm{R}=$ valeur des coefficients de corrélation canonique; $(36,172)$ : Nombre de degrés de liberté pour le test F 
Tableau 3. Coefficients de corrélation entre les nouvelles variables canoniques et les anciennes variables (précipitations et indices climatiques) dans le bassin versant de la rivière Saint-François (1914-1990).

Table 3. Canonical correlation coefficients between the new canonical variables and the former variables (precipitation and climatic oscillations) for the Saint-François River watershed (1916-1988).

\begin{tabular}{|c|c|c|c|c|c|c|}
\hline & V1 & V2 & V3 & W1 & W2 & W3 \\
\hline Disraeli & 0,2492 & 0,8833 & 0,3972 & & & \\
\hline Drummondville & $-0,4557$ & 0,0710 & 0,8873 & & & \\
\hline Sherbrooke & 0,8686 & $-0,0161$ & 0,4953 & & & \\
\hline ONAa & & & & $-0,1584$ & $-0,4301$ & 0,5031 \\
\hline ONAh & & & & $-0,2139$ & 0,1601 & 0,8020 \\
\hline ONAe & & & & $-0,0483$ & $-0,6566$ & 0,1150 \\
\hline $\mathrm{OAa}$ & & & & 0,3387 & 0,2186 & 0,5193 \\
\hline OAh & & & & $-0,2260$ & 0,3369 & 0,6403 \\
\hline OAe & & & & 0,8314 & $-0,2872$ & 0,0883 \\
\hline AUSa & & & & $-0,0997$ & 0,0394 & 0,1200 \\
\hline AUSh & & & & $-0,0620$ & 0,0800 & 0,2646 \\
\hline AUSe & & & & $-0,1586$ & 0,0349 & $-0,0026$ \\
\hline NIN3a & & & & $-0,4096$ & 0,0712 & $-0,1989$ \\
\hline NIN3h & & & & $-0,3666$ & 0,1033 & $-0,1627$ \\
\hline NIN3e & & & & $-0,4103$ & 0,0379 & $-0,2307$ \\
\hline
\end{tabular}

$\mathrm{a}=$ indice annuel; $\mathrm{h}=$ indice hivernal; $\mathrm{e}=$ indice estival. Les valeurs significatives des coefficients de corrélation les plus élevées apparaissent en gras.

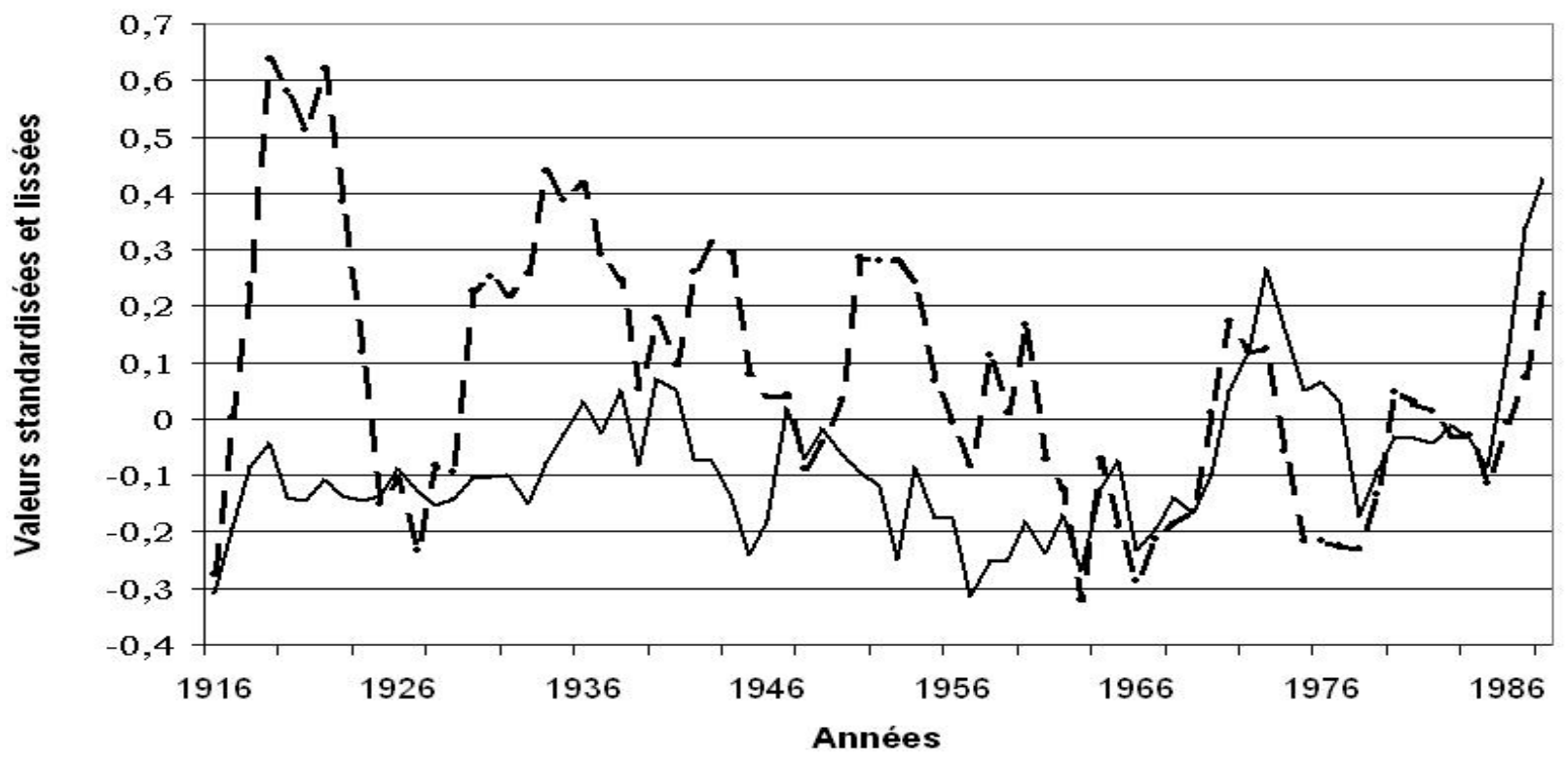

Figure 4. Valeurs normalisées et lissées des indices climatiques OA (ligne continue) et ONA (ligne discontinue). Normalized and five-year running means of annual $O A$ (full line) and NAO (broken line) indexes values (1914-1990). 
Tableau 4. Coefficients de corrélation canonique calculés durant les périodes 1914-1950 et 1951-1990.

Table 4. Canonical correlation coefficients calcuted for the periods 1914-1950 and 1951-1990.

\begin{tabular}{ccccccccc}
\hline & \multicolumn{3}{c}{ Période 1914-1950 } & & \multicolumn{5}{c}{ Période 1951-1990 } \\
\hline & $\mathrm{R}$ & $\mathrm{VP}$ & $\mathrm{F}$ & $\mathrm{Pr}>\mathrm{F}$ & $\mathrm{R}$ & $\mathrm{VP}$ & $\mathrm{F}$ & $\mathrm{pr}>\mathrm{F}$ \\
$\lambda_{1}$ & 0,979 & 23,31 & 11,29 & $<\mathbf{0 , 0 0 0 1}$ & 0,942 & 7,94 & 7,27 & $<\mathbf{0 , 0 0 0 1}$ \\
$\lambda_{2}$ & 0,949 & 9,15 & 6,13 & $<\mathbf{0 , 0 0 0 1}$ & 0,877 & 3,34 & 5,25 & $<\mathbf{0 , 0 0 0 1}$ \\
$\lambda_{3}$ & 0,748 & 0,748 & 1,65 & 0,1582 & 0,791 & 1,68 & 4,19 & $\mathbf{0 , 0 0 1 7}$ \\
\hline
\end{tabular}

$\mathrm{VP}=$ Valeur propre; $\mathrm{F}=$ test de $\mathrm{F}$ de Fisher-Snedecor.

Tableau 5. Coefficients de corrélation entre les nouvelles variables canoniques et les précipitations mesurées aux trois stations.

Table 5. Canonical correlation coefficients between the new canonical variables and precipitation at the three sampling stations.

\begin{tabular}{lcccccc}
\hline Stations & \multicolumn{3}{c}{ Période 1914-1950 } & \multicolumn{3}{c}{ Période 1951-1990 } \\
\hline \multirow{2}{*}{ V1 } & V2 & V3 & V1 & V2 & V3 \\
Disraeli & 0,0118 & $\mathbf{0 , 7 8 4 9}$ & 0,6195 & 0,0413 & $\mathbf{0 , 9 9 5 7}$ & $-0,0835$ \\
Drummondville & $\mathbf{- 0 , 8 2 0 7}$ & $-0,2779$ & 0,4993 & 0,0162 & $\mathbf{0 , 8 1 9 5}$ & 0,5728 \\
Sherbrooke & $\mathbf{0 , 9 7 9 1}$ & $-0,1329$ & 0,1540 & $-0,5665$ & $\mathbf{0 , 7 8 0 6}$ & 0,2643 \\
\hline
\end{tabular}

Drummondville correspond à l'épisode humide à Sherbrooke. En revanche, après la période 1950, les trois stations sont positivement corrélées à la seconde variable canonique confirmant aussi le synchronisme observé dans la succession des épisodes secs et humides dans le bassin versant après cette date.

Quant à la relation entre les nouvelles variables canoniques et les indices climatiques (Tableau 6), aussi bien avant qu'après la période 1950, c'est l'oscillation arctique qui présente les valeurs des coefficients de corrélation les plus élevées avec les nouvelles variables canoniques. Avant 1950, OA est corrélée positivement à la première variable canonique (W1) en été mais négativement en hiver. Il s'ensuit qu'avant 1950, la corrélation entre les précipitations mesurées à Sherbrooke et cet indice est positive en été, mais négative en hiver. C'est l'inverse à Drummondville. Quant à l'oscillation nord-atlantique (ONA), elle est corrélée à la seconde nouvelle variable canonique (W2). Par conséquent, cet indice est négativement corrélé aux précipitations mesurées à la station de Disraeli. Son influence semble se limiter seulement au nord du bassin versant. On notera aussi que le lien entre les indices climatiques et les précipitations est plus fort aux stations de Sherbrooke et Drummondville (axe Est-Ouest) qu'à la station de Disraeli. Après 1950, OA et ONA sont positivement corrélées aux précipitations mesurées aux trois stations. Ce lien est compatible avec le synchronisme observé dans la variabilité interannuelle de ces deux indices climatiques (Figure 4). Le fait le plus important à souligner est la diminution des valeurs des coefficients de corrélation entre les nouvelles variables canoniques et certains indices climatiques, en particulier l'oscillation arctique, après 1950. Enfin, comme nous l'avons déjà mentionné, les indices hivernaux de OA et ONA sont corrélés aussi bien avant qu'après 1950. Il devient important de vérifier si ces deux indices peuvent servir à prédire les périodes sèches et humides aux trois stations analysées.

\subsection{Relation entre les précipitations annuelles et les indices climatiques pendant les périodes sèches et humides}

Nous avons corrélé les indices climatiques et les précipitations avant et après 1950 d'une part, et avant et après 1970, d'autre part. Les résultats sont résumés dans le tableau 7. Il ressort de ce tableau les faits saillants suivants :

- Avant 1950, pendant la période sèche, la station de Sherbrooke est positivement corrélée à OA mais négativement corrélée à ONA et NIN3. Pendant la période humide, la station est corrélée positivement 
Tableau 6. Coefficients de corrélation entre les nouvelles variables canoniques et les indices climatiques.

Table 6.c Canonical correlation coefficients between the new canonical variables and climatic oscillations.

\begin{tabular}{|c|c|c|c|c|c|c|}
\hline & \multicolumn{3}{|c|}{ Période 1914-1950 } & \multicolumn{3}{|c|}{ Période 1951-1990 } \\
\hline & W1 & W2 & W3 & W1 & W2 & W3 \\
\hline $\mathrm{OAa}$ & 0,4957 & 0,1756 & 0,4536 & $-0,0748$ & 0,4708 & 0,4679 \\
\hline OAh & $-0,6411$ & 0,4310 & 0,2679 & 0,0647 & 0,4960 & 0,4661 \\
\hline OAe & 0,9631 & $-0,2110$ & 0,0387 & $-0,3086$ & 0,1818 & 0,5675 \\
\hline ONAa & $-0,0608$ & $-0,5691$ & 0,5830 & 0,1308 & 0,0869 & 0,0307 \\
\hline ONAh & $-0,5698$ & $-0,0489$ & 0,4547 & 0,1120 & 0,5579 & 0,4332 \\
\hline ONAe & 0,1996 & $-0,6717$ & 0,4190 & 0,0287 & $-0,4010$ & $-0,3383$ \\
\hline AUSa & $-0,5426$ & 0,2093 & $-0,0346$ & $-0,5568$ & 0,0499 & $-0,2885$ \\
\hline AUSh & $-0,5260$ & 0,3556 & 0,2650 & $-0,5597$ & 0,1485 & $-0,2656$ \\
\hline AUSe & $-0,4703$ & 0,0869 & $-0,1779$ & $-0,4582$ & $-0,0321$ & $-0,2759$ \\
\hline NIN3a & $-0,3470$ & 0,2527 & 0,1278 & 0,4509 & $-0,3013$ & 0,0274 \\
\hline NIN3h & $-0,3183$ & 0,2374 & 0,0921 & 0,4273 & $-0,2129$ & $-0,0194$ \\
\hline NIN3e & $-0,3351$ & 0,2605 & 0,1572 & 0,4397 & $-0,3597$ & 0,0570 \\
\hline
\end{tabular}

Tableau 7. Indices climatiques influençant la variabilité interannuelle des précipitations dans le bassin versant de la rivière Saint-François avant et après 1935 et 1970.

Table 7. Relationships between climatic oscillations and precipitations before and after 1935 and 1970 in the SaintFrançois River watershed.

\begin{tabular}{|c|c|c|c|c|c|c|c|}
\hline & & \multicolumn{2}{|c|}{ Disraeli } & \multicolumn{2}{|c|}{ Drummondville } & \multicolumn{2}{|c|}{ Sherbrooke } \\
\hline & & Période & $\begin{array}{c}\text { Indices } \\
\text { climatiques }\end{array}$ & Période & $\begin{array}{c}\text { Indices } \\
\text { climatiques }\end{array}$ & Période & $\begin{array}{c}\text { Indices } \\
\text { climatiques }\end{array}$ \\
\hline \multirow{2}{*}{$\begin{array}{l}\text { Avant } \\
1950\end{array}$} & $\begin{array}{c}\text { Avant } \\
1935\end{array}$ & Sèche & $\begin{array}{c}\text { OA (-) } \\
\text { NIN3(+) }\end{array}$ & Humide & $\begin{array}{c}\text { OA (-) ONA } \\
(+)\end{array}$ & Sèche & $\begin{array}{c}\text { OA (+), NIN3 } \\
(-) \text {, ONA }(-)\end{array}$ \\
\hline & $\begin{array}{c}\text { Après } \\
1935\end{array}$ & Sèche/humide & $\begin{array}{c}\mathrm{OA}(-) \text { AUS } \\
(+)\end{array}$ & Sèche & $\begin{array}{c}\mathrm{OA}(-) \text { AUS } \\
(+)\end{array}$ & Humide & $\mathrm{ONA}(+)$ \\
\hline \multirow{2}{*}{$\begin{array}{l}\text { Après } \\
1950\end{array}$} & $\begin{array}{c}\text { Avant } \\
1970\end{array}$ & Sèche & $\begin{array}{l}\text { ONA (+) OA } \\
(+) \text { NIN3 }(-)\end{array}$ & Sèche & - & Sèche & $\mathrm{AO}(+)$ \\
\hline & $\begin{array}{c}\text { Après } \\
1970\end{array}$ & Humide & $\begin{array}{c}\text { OA (-) } \\
\text { ONA (-) }\end{array}$ & Humide & ONA $(+)$ & Humide & $\begin{array}{l}\operatorname{AUS}(+), \\
\text { NIN (-) }\end{array}$ \\
\hline
\end{tabular}

à ONA. Quant à la station de Drummondville, elle est négativement corrélée à $\mathrm{OA}$ mais positivement à $\mathrm{ONA}$ pendant la période humide. En période sèche, elle est toujours négativement corrélée à $\mathrm{OA}$ mais positivement corrélée à AUS. Enfin, la station de Disraeli est comparable à celle de Drummondville. Toutefois, la station n'est pas significativement corrélée à ONA mais elle devient positivement corrélée à NIN3.
- Après 1950, la station de Sherbrooke est toujours corrélée positivement à $\mathrm{OA}$ pendant la période de sécheresse. Mais cette corrélation disparaît pendant la période humide où la station devient corrélée aux indices AUS et NIN3. Pendant la période sèche, la station de Drummondville n'est plus significativement corrélée à aucun indice alors que pendant la période humide, elle est positivement corrélée à ONA. Quant à la station de Disraeli, durant 
l'épisode sec, elle est positivement corrélée à ONA et OA mais négativement à NIN3. En revanche, pendant la période humide, la station est négativement corrélée aux deux premiers indices.

\section{DISCUSSION ET CONCLUSION}

Le lissage au moyen des moyennes mobiles glissantes et la standardisation des précipitations annuelles dans le bassin versant de la rivière Saint-François pendant la période 1914-1990 ont mis en évidence deux types de changements :

- Le premier type de changement, survenu autour de 1950, est un changement qui affecte la répartition spatiale des précipitations dans tout le bassin versant. En effet, avant 1950, la variabilité interannuelle des précipitations n'était pas synchrone dans tout le bassin versant. Ainsi, par exemple, durant la période 1914-1935, les précipitations étaient déficitaires (période sèche) à la station de Sherbrooke mais excédentaire (période humide) à la station de Drummondville. Mais après 1950, la variabilité interannuelle des précipitations était devenue synchrone. La période sèche de 1951-1969 et celle humide de 1971-1985 étaient observées à toutes les stations du bassin versant de la rivière Saint-François. Sans toutefois préciser la nature du changement comme nous venons de le faire, COULIBALY et BURN $(2004,2005)$ étaient les premiers à détecter un changement autour de l'année 1950 sur les séries des débits moyens annuels et saisonniers au Canada. Cependant, ils n'ont pas précisé la cause (facteur) de ce changement.

- Le second type de changement est survenu autour des années 1935 et 1970. Il correspond à un changement des totaux pluviométriques. Ainsi, ces deux dates marquent le passage d'une période sèche à une période humide et vice versa selon les stations. Le changement de 1970 a été observé dans de nombreuses séries hydroclimatiques au moyen de plusieurs méthodes. ANCTIL et COULIBALY (2004) ainsi que COULIBALY et BURN (2004) ont détecté un changement en 1970 sur les séries des débits moyens annuels au Québec et dans le reste du Canada au moyen de l'analyse des ondelettes. Quant à SHABBAR et al. (1997b), ils ont aussi observé le même changement sur les séries des températures hivernales des stations de la côte orientale du Canada et occidentale du Groenland. Le changement de 1970 a été aussi observé dans d'autres régions de l'hémisphère Nord, notamment en Irlande (KIELY, 1999) et aux États-Unis d'Amérique (CHEN et RAO, 2002). Mais en ce qui concerne le changement survenu autour de 1935, aucune étude ne l'a jamais encore détecté au Canada. Enfin, le synchronisme des périodes humides observées aux trois stations après 1970 peut aisément expliquer l'augmentation de la fréquence et de l'intensité des inondations observées dans le bassin versant durant la décennie 70 car le ruissellement, générateur des crues, se produit à l'échelle de tout le bassin versant.

L'analyse de corrélations canoniques a révélé un lien relativement fort entre les indices climatiques et les précipitations dans le bassin versant de la rivière SaintFrançois. Mais ce lien est influencé par les deux types de changements qui ont affecté la variabilité interannuelle des précipitations.

- Si on considère toute la période d'analyse (1914-1990), les deux indices climatiques, qui sont significativement corrélés aux précipitations, sont l'oscillation arctique (OA) et l'oscillation nord-atlantique (ONA). Le premier indice est corrélé positivement aux stations de Sherbrooke et de Drummondville alors que le second indice l'est positivement à la station de Drummondville mais négativement à celle de Disraeli.

- Avant la période 1950, OA est positivement corrélée en été mais négativement en hiver aux stations de Sherbrooke et de Drummondville. ONA est négativement corrélée à la station de Disraeli. Après 1950, l'influence de ces deux indices s'étend sur l'ensemble du bassin versant et leur variabilité interannuelle devient quasi synchrone. Ce qui expliquerait le synchronisme de la variabilité interannuelle des précipitations observé après cette date. Les deux indices sont positivement corrélés aux précipitations mais les valeurs des coefficients de corrélation diminuent par rapport à la période avant 1950 .

- L'analyse des corrélations entre les périodes sèches et humides et les indices climatiques n'a révélé aucune constance dans la relation entre les indices et ces périodes. Toutefois, ONA est toujours corrélée positivement à la station de Drummondville pendant les périodes humides tandis que OA l'est à la station de Sherbrooke pendant les périodes sèches. Il s'ensuit que cet indice permet de prédire les périodes sèches à la station de Sherbrooke alors que ONA, les périodes humides à la station de Drummondville.

- Il existe une corrélation entre OA et ONA en hiver avant et après 1950 comme l'avait déjà mentionné WALLACE (2000). En été, les deux indices ne sont plus corrélés. Ce qui nous permet d'affirmer que ces indices représenteraient deux phénomènes climatiques différents dont les effets peuvent se superposer en hiver mais s'opposer en été.

Dans leur article de synthèse, KINGSTON et al. (2006) ont tenté d'expliquer les mécanismes climatiques par lesquels OA et ONA influencent la circulation générale au-dessus de la partie Nord-Est du continent nord américain. En effet, lorsque les deux indices climatiques sont en mode positif, on observe une hausse de fréquence de l'air froid polaire dans les régions 
Nord-Est et Est du Canada provoquant ainsi une diminution de la température et des précipitations. En revanche, dans la région Nord-Est des États-Unis d'Amérique, on observe une hausse de fréquence de masse d'air chaud provenant du Sud. Ceci entraîne une hausse de la température et des précipitations. À l'échelle du bassin versant de la rivière SaintFrançois, lorsqu'on considère toute la période d'analyse, OA est positivement corrélée aux précipitations comme dans le NordEst des États-Unis d'Amérique. Il en résulte une hausse de la fréquence des masses d'air provenant du Sud en hiver dans le bassin versant lorsque OA est en mode positif. Comme nous l'avons déjà mentionné, ce sont ces masses d'air qui apportent une quantité plus importante des précipitations dans le bassin versant, particulièrement en été. Cependant, il faut noter que le lien entre $\mathrm{OA}$ et les précipitations change dans le temps. Ce changement a été observé surtout après 1950. Après cette date, l'indice n'est plus significativement corrélé aux précipitations. De même, dans le Nord-Est des États-Unis, on n'a observé aucun lien significatif entre les précipitations et ONA, un indice corrélé à OA, pendant la même période (KINGSTON et al., 2006). Si on considère les périodes sèches et humides, on observe que OA peut être positivement ou négativement corrélée aux périodes sèches selon les stations. Ainsi, il n'existe aucune cohérence spatiale du lien entre les périodes de sécheresse/humidité et OA. Ce qui limite l'utilisation de OA pour prédire la succession des périodes sèches et humides dans le bassin versant de la rivière Saint-François. Toutefois, à la station de Sherbrooke, OA est toujours associée aux épisodes de sécheresse. L'indice servirait ainsi à prédire les périodes de sécheresse à cette station.

Quoi qu'il en soit, il ressort de cette étude que OA apparaît comme le principal facteur de variabilité temporelle des précipitations dans le bassin versant de la rivière Saint-François. Toutefois, cet indice ne permet que de prédire les périodes sèches à la station de Sherbrooke. Mais si on tient compte de ONA, qui lui est corrélée en hiver, il devient possible de prédire la succession des périodes sèches et humides dans tout le bassin versant en raison du synchronisme des périodes humides et sèches observées après 1950.

\section{RÉFÉRENCES BIBLIOGRAPHIQUES}

AFIFI A.A. et V. CLARK (1996). Computer-aided multivariate analysis. $3^{\text {rd }}$ edition. Chapman and Hall (Éditeurs), New York, 505p.

AMBAUM M.H.P., B.J. HOSKINS et D.B. STEPHENSON (2001). Arctic oscillation or north atlantic oscillation? J. Clim., 15, 3495-3507.
ANCTIL F. et P. COULIBALY (2004). Wavelet analysis of the interannual variability in southern Québec streamflow. J. Clim., 17, 163-73.

ASSANI A.A. (1999). Analyse de la variabilité temporelle des précipitations (1916-1996) à Lubumbashi (CongoKinshasa) en relation avec certains indicateurs de la circulation atmosphérique (oscillation australe) et océanique (El Nino/La Nina). Sécheresse 10, 245-252.

BALDWIN M.P. et T.J. DUNKERTON (1999). Propagation of the Arctic oscillation from the stratopshere to the troposphere. J. Geophys. Res. 104, 30937-30946.

BERRI G.J. et G.I. BERTOSA (2004). The influence of tropical Atlantic and Pacific oceans on precipitations variability over southern central South America on seasonal time scales. Intern. J. Climatol., 24, 415-435.

BRADBURY J.A., S.L. DINGMAN et B.D. KEIM (2002). New England drought and relations with large scale atmospheric circulation patterns. J. Amer. Water Res. Ass., 38, 1287-1299.

BROWN R.D. et B.E. GOODISON (1996). Interannual variability in reconstructed Canadian snow cover 1915-1992. J. Clim., 9, 1299-1318.

CADET D. et R. GARNIER (1988). L'oscillation australe et ses relations avec les anomalies climatiques globales. $\mathrm{La}$ Météorologie, 21, 4-18.

CHEND et Y. CHEN (2003). Association between winter temperature in China and upper air circulation over East Asia revealed by canonical correlation analysis. Global Plan., 37, 315-325.

CHEN H.L. et A.R. RAO (2002). Testing hydrologic time series for stationarity. J. Hydrol. Eng., 7, 129-36.

COULIBALY P. et D.H. BURN (2004). Walevet analysis of variability in annual Canadian streamflows. Water Res., 40, 1-14.

COULIBALY P. et D.H. BURN (2005). Spatial and temporal variability of canadian seasonal streamflows. J. Clim., 18, 191-210.

COULIBALY P. F. ANCTIL, P. RASMUSSEN et B. BOBÉE (2000). A recurrent neural netwoks approach using indices of low-frequency climatic variability to forecast regional annual runoff. Hydrol. Process. 14, 2755-77. 
DÉRY S.J., M. STIEGLITZ, E.C. McKENNA et E.F. WOOD (2005). Characteristics and trends of river discharge into Hudson, James, and Ungava Bays, 19642000. J. Clim., 18, 2540-2557.

DÉRY S.J. et E.F. WOOD (2004). Teleconnection between the arctic oscillation and hudson bay river discharge. Geophys. Res. Lett., 31, LI18205, doi : 1029/2004GL020729.

DÉRY S.J. et E.F. WOOD (2005). Decreasing river in northern Canada. Geophys. Res. Lett., 32, L10401, doi: 10.1029/2005GL022845.

DESER C. (2000). On the teleconnectivity of the "Arctic Oscillation." Geophys. Res. Lett., 27, 779-782.

DÜNKELOH A. et J. JACOBEIT (2003). Circulation dynamics of Mediterranean precipitation variability 194898. Intern. J. Climatol., 23, 1843-1866.

ENVIRONNEMENT CANADA (2003). Adjusted historical canadian climate data (AHCCD). Climate research branch, meteorological service, Canada. (http://www.cccma.bc.ec. gc.ca/ahccd/).

GROISMAN P.Y. et D.R. EASTERLING (1994). Variability and trends of total precipitation and snowfall over United States and Canada. J. Clim., 7, 184-205.

HAYLOCK M.R. et C.M. GOODESS (2004). Interannual variability of European extreme winter rainfall and links with mean large-scale circulation. Intern. J. Climatol., 24, 759-776

HIGGINS R.W., A. LEETMAA, Y. XUE et A. BARNSTON (2000). Dominant factors influencing the seasonal predictability of U.S precipitation and surface air temperature. J. Clim., 13, 3994-4017.

HIGUSHI K., C.W. YUEN et A. SHABBAR (2000). Ice storm'98 in southcentral Canada and northeasthern Unites States: A climatological perspective. Theor. Appl. Climatol., 66, 61-79.

JAIN S., M. HOERLING et J. EISCHEID (2005). Decreasing reliability and increasing synchroneity of western north american streamflow. J. Clim., 18, 613-618.

JONES N.K. (2004). An analysis of the Massawippi floods of 1982 and 1994. Can. Water Res. J., 29, 73-84.

KINGSTON D.G., D.M. LAWLER et G.R. McGREGOR
(2006). Linkage between atmospheric circulation, climate and streamflow in the northern Atlantic: research prospects. Progr. Phys. Geogr., 30, 143-174.

LABAT D. (2005). Recent advances in wavelet analyses: part I. A review of concepts. J. Hydrol., 314, 275-288.

LOLIS C.J., A. BARTZOKAS et B.D. KATSOULIS (2004). Relation between sensible and latent heat fluxes in the Mediterranean and precipitation in the Greek area during winter. Intern. J. Climatol., 24, 1803-1816.

MEKIS E. et W. HOGG (1999). Rehabilitation and analysis of canadian daily precipitation time series. AtmosphereOcean, 37, 53-85.

PROULX H., G. JACQUES, A.M. LAMOTHE et J. LITYNSKI (1987). Climatologie du Québec méridional. Environnement Québec, Québec, rapport MP-65.

RIGOR I.G., J.M. WALLACE et R.L. COLONY (2002). Response of sea ice to the Arctic oscillation. J. Clim., 15, 2648-2663.

ROGERS J.C. (1984). The association between the north atlantic oscillation and the southern oscillation in the northern hemisphere. Month. Weather Rev., 112, 1999-2015.

ROPELEWSKI, C.F. et M.S. HALPERT (1987). Global and regional scale precipitation patterns associated with the El Niño/Southern Oscillation. Mon. Weather Rev., 115, 1606-1626.

SAINT-LAURENT D., COUTURE C. et E. MCNEIL (2001). Spatio-temporal analysis of floods of the SaintFrançois drainage basin, Québec, Canada. Environments, 29, 74-90.

SHABBAR A. et B. BONSAL (2004). Associations between low frequency variability modes and winter temperature extremes in Canada. Atmopshere-Ocean, 42, 127-140.

SHABBAR A., B. BONSAL et M. KHANDEKAR (1997). Canadian precipitation patterns associated with the Southern Oscillation. J. Clim., 10, 3016-27.

SHABBAR A., K. HIGUCHI, W. SKINNER et J.L. KNOX (1997). The association between the BWA index and winter surface temperature variability over eastern Canada and west Greenland. Intern. J. Clim., 17, 1195-1210. 
SHABBAR A. et W. SKINNER (2004). Summer drought patterns in Canada and the relationship to global sea surface temperatures. J. Clim., 17, 2866-2880.

SHERIDAN S.C. (2002). The redevelopment of a weathertype classification scheme for North America. Inter. J. Clim., 25, 51-68.

SHERIDAN S.C. (2003). North America weather-type frequency and teleconnection indices. Intern. J. Clim., 23, $27-45$.

SIEW-YAN-YU T.O., J. ROUSSELLE, G. JACQUES et V.T.V. NGUYEN (1998). Régionalisation du régime des précipitations dans la région des Bois-Francs et de l'Estrie par l'analyse en composantes principales. Can. J. Civ. Eng., 25, 105-1058.

STONE, D.A, WEAVER A.J. et F. ZWIERS (2000). Trends in Canadian precipitation intensity. Atmosphere-Ocean, 38, 321-347

THOMPSON D.W.J. et J.M WALLACE (1998). The Arctic oscillation signature in the wintertime geopotential height and temperature fields. Geophys. Res. Lett., 25, $1297-$ 1300.

THOMPSON D.W.J. et J.M. WALLACE (2001). Regional climate impacts of the northern hemisphere annular mode. Science, 293, 85-89.

WALLACE J.M. (2000). North atlantic oscillation/annular mode: two paradigms-one phenomenon. Quart. J. Roy. Meteor. Soc., 126, 791-805.

WALLACE J.M. et D.W.J. THOMPSON (2002). The Pacific center of action of the northern hemisphere annular mode: real or artifact? J. Clim., 15, 1987-1991

WANNER H. (1999). Le balancier de l'Atlantique nord. La Recherche, 321, 72-73. 